\title{
There is a Close Association Between the Recovery of Liver Injury and Glycemic Control after SGLT2 Inhibitor Treatment in Japanese Subjects with Type 2 Diabetes: A Retrospective Clinical Study
}

\author{
Tomoe Kinoshita - Masashi Shimoda (D) · Junpei Sanada · Yoshiro Fushimi • \\ Yurie Hirata $\cdot$ Shintaro Irie - Atsushi Obata - Tomohiko Kimura - Hidenori Hirukawa • \\ Kenji Kohara · Fuminori Tatsumi · Shinji Kamei · Shuhei Nakanishi • \\ Tomoatsu Mune $\cdot$ Kohei Kaku $\cdot$ Hideaki Kaneto
}

Received: March 7, 2018 / Published online: June 21, 2018

(c) The Author(s) 2018

\section{ABSTRACT}

Introduction: Sodium-glucose co-transporter 2 (SGLT2) inhibitors function not only to reduce hyperglycemia but also to ameliorate liver injury and reduce body weight. The aim of this study was to examine in which subjects SGLT2 inhibitors are more effective for glycemic control, liver injury, and obesity in Japanese subjects with type 2 diabetes mellitus.

Methods: We enrolled a total of 156 subjects with type 2 diabetes who initiated SGLT2 inhibitor treatment after September 1, 2014 in Kawasaki Medical School (Protocol No. 2375). We evaluated the alteration of glycemic control,

Enhanced digital features To view enhanced digital features for this article go to https://doi.org/10.6084/ m9.figshare.6270008.

T. Kinoshita $\cdot$ M. Shimoda $(\bowtie) \cdot$ J. Sanada

Y. Fushimi · Y. Hirata - S. Irie - A. Obata .

T. Kimura · H. Hirukawa - K. Kohara - F. Tatsumi ·

S. Kamei · S. Nakanishi · T. Mune · H. Kaneto

Division of Diabetes, Metabolism and

Endocrinology, Kawasaki Medical School, Kurashiki, Japan

e-mail: masashi-s@med.kawasaki-m.ac.jp

K. Kaku

Division of General Internal Medicine, Kawasaki

Medical School, Kurashiki, Japan liver injury, body mass composition, and various clinical parameters.

Results: SGLT2 inhibitors significantly ameliorated glycemic control and improved liver injury in Japanese subjects with type 2 diabetes. SGLT2 inhibitors were more effective for liver injury when glycemic control was improved with SGLT2 inhibitors. In multivariate analyses, the amelioration of glycemic control was an independent determinant factor for the improvement of liver damage in Japanese subjects with type 2 diabetes. The reverse was also correct; the improvement of liver damage was an independent determinant factor for the amelioration of glycemic control.

Conclusion: Recovery of liver injury with SGLT2 inhibitor treatment was closely associated with their effects on glycemic control in Japanese subjects with type 2 diabetes.

Keywords: Liver injury; SGLT2 inhibitor; Type 2 diabetes mellitus

\section{INTRODUCTION}

Sodium-glucose co-transporter 2 (SGLT2) inhibitors exert beneficial effects by reducing renal glucose reabsorption, which leads to excess glucose excretion and calorie loss [1-7]. SGLT2 
inhibitors function to lower blood glucose levels in an insulin-independent manner. While overweight and subsequent liver damage such as fatty liver are often also present in subjects with type 2 diabetes, SGLT2 inhibitors function to reduce body weight and ameliorate liver injury in addition to the improvement of glycemic control [1-7]. SGLT2 inhibitors also have a multilateral action such as the improvement of the lipid metabolism and decreased of blood pressure and serum uric acid level [8]. It has been also reported that SGLT2 inhibitors reduce insulin resistance and preserve pancreatic $\beta$-cell function, which ultimately delays the worsening of pathogenesis of type 2 diabetes [9-15]. Furthermore, EMPA-REG OUTCOME clinical trials showed that subjects with type 2 diabetes at high risk for cardiovascular events who received empagliflozin had a lower rate of the primary composite cardiovascular outcome and of death from any cause compared to the subjects receiving placebo [16-19]. Subanalyses in the same clinical trials showed that in subjects with type 2 diabetes at high cardiovascular risk, empagliflozin was associated with slower progression of kidney disease and lower rates of clinically relevant renal events [20]. Therefore, SGLT2 inhibitors have been drawing much attention. In clinical practical, however, SGLT2 inhibitors do not necessarily reduce body weight and do not necessarily ameliorate liver injury and/or glycemic control in all subjects with type 2 diabetes. On the basis of these phenomena, we decided to start this clinical study.

The primary outcome was change of liver injury, and the secondary outcome was change of HbA1c. Furthermore, we evaluated the correlation between change of liver injury or HbA1c and clinical background.

\section{METHODS}

\section{Subjects}

We examined a total of 156 subjects with type 2 diabetes who visited the Department of Diabetes, Endocrinology, and Metabolism, Kawasaki Medical School from September, 2014 to
August, 2016, and started taking SGLT2 inhibitors. SGLT2 inhibitor (luseogliflozin (52\%), tofogliflozin (29\%), ipragliflozin $(15 \%)$, or dapagliflozin (4\%)) was added to existing drugs. It is noted that these four drugs are commercially available in everyday practical medicine. The baseline drugs were maintained in the same dose during the observation period. We excluded the subjects with renal dysfunction (eGFR $<45 \mathrm{~mL} / \mathrm{min}$ ), cardiac failure, liver disease (viral hepatitis, alcoholic hepatitis, autoimmune liver disease, or liver cirrhosis) and use of steroids. The study protocol was approved by the hospital ethics committee (No. 2375). All procedures performed in studies involving human participants were in accordance with the ethical standards of the institutional and/or national research committee and with the 1964 Declaration of Helsinki and its later amendments or comparable ethical standards and Good Clinical Practice. Informed consent was obtained by opt out.

We measured common clinical parameters, including height, body mass index (BMI) (kg/ $\mathrm{m}^{2}$ ), and blood pressure. Also, we examined various parameters such as HbA1c, fasting plasma glucose (FPG) or postprandial plasma glucose (PPG), low-density lipoprotein (LDL) cholesterol, high-density lipoprotein (HDL) cholesterol, triglyceride (TG), non-esterified fatty acid (NEFA), creatinine (Crn), estimated glomerular filtration rate (eGFR), uric acid, aspartate aminotransferase (AST), alanine aminotransferase (ALT), and gamma-glutamyl transpeptidase $(\gamma$-GTP). The data related to immunoreactive insulin (IRI), homeostasis model assessment of insulin resistance (HOMAIR), and NEFA were those of subjects with fasting blood sampling. Visceral fat area and skeletal muscle mass were calculated using a bioelectrical impedance body composition analyzer, InBody 770 (InBody Japan Inc.).

\section{Statistical Analyses}

All analyses were performed by using JMP version 9. Wilcoxon signed-rank test was used for the comparison between two paired groups. Spearman rank correlation analysis was 
performed to examine the association between ALT, HbA1c, BMI, and various clinical parameters. Concerning parameters with non-normal distribution, various analyses were performed after Box-Cox Y conversion. The results were expressed as median (interquartile range) for non-parametric variables. Throughout the statistical analysis, $p<0.05$ was considered significant.

\section{RESULTS}

\section{Clinical Characteristics of Study Subjects}

As shown in Table 1, clinical characteristics of all subjects $(n=156)$ in this study at baseline were as follows: men/women 76:80; age, 54 years old (median); duration of diabetes, 8 years; HbA1c, $7.5 \%$; fasting plasma glucose (FPG), $138 \mathrm{mg} / \mathrm{dL}$; postprandial plasma glucose (PPG), $186 \mathrm{mg} / \mathrm{dL}$; urinary glucose, $0.07 \mathrm{~g} /$ g Crn; body weight, $78.5 \mathrm{~kg} ; \mathrm{BMI}, 29.6 \mathrm{~kg} / \mathrm{m}^{2}$; visceral fat area, $140 \mathrm{~cm}^{2}(n=108)$; skeletal muscle mass, $26.7 \mathrm{~kg}(n=108)$. Other characteristics of the subjects in this study were as follows: LDL cholesterol (LDL-Chol), $100 \mathrm{mg}$ / dL; HDL cholesterol (HDL-Chol), $47 \mathrm{mg} / \mathrm{dL}$; triglyceride, $129 \mathrm{mg} / \mathrm{dL}$; systolic blood pressure, $130 \mathrm{mmHg}$; diastolic blood pressure, $80 \mathrm{mmHg}$; ALT, $34 \mathrm{U} / \mathrm{L} ;$ AST, $26 \mathrm{U} / \mathrm{L} ; \gamma$-GTP, $38 \mathrm{U} / \mathrm{L}$; Crn, $0.60 \mathrm{mg} / \mathrm{dL}$; eGFR, $95.2 \mathrm{~mL} / \mathrm{min} / 1.73 \mathrm{~m}^{2}$; uric acid (UA), $5.4 \mathrm{mg} / \mathrm{dL}$; urinary albumin excretion, $22.6 \mathrm{mg} / \mathrm{g}$ Crn.

The proportions of subjects with diabetic neuropathy, retinopathy, nephropathy, cerebrovascular disorder, and ischemic heart disease were $28 \%, 14 \%, 33 \%, 4 \%$, and $5 \%$, respectively. The therapeutic situation at administration was as follows: insulin, 14\%; glucagon-like peptide1 (GLP-1) receptor activator, 10\%; dipeptidyl peptidase-4 (DPP-4) inhibitor, 62\%; sulfonylurea (SU), 24\%; glinide, $8 \%$; biguanide, 76\%; thiazolidinediones, $37 \%$; $\alpha$-glucosidase inhibitor $(\alpha-G I), 12 \%$. Other medications were as follows: angiotensin II receptor blocker (ARB), 38\%; calcium channel blocker (CCB), 23\%; diuretic, 5\%; statin, 65\%; fibrate, 10\%.
Efficacy of SGLT2 Inhibitor on Various Metabolic Parameters Including Body Weight, Glycemic Control, and Liver Damage

Twelve weeks after starting SGLT2 inhibitor, body weight and BMI were significantly reduced compared to before the treatment $(p<0.0001)$. Reduction from baseline body weight and BMI was $-2.8 \mathrm{~kg}$ and $-0.9 \mathrm{~kg} / \mathrm{m}^{2}$, respectively. HbA1c and blood glucose levels were also significantly decreased. Reduction from baseline HbA1c, FPG, and PPG was $-0.4 \%,-15$ and $-27 \mathrm{mg} / \mathrm{dL}$, respectively. Liver injury was also significantly improved; 12 weeks after the initiation of SGLT2 inhibitor, ALT, AST, and $\gamma$-GTP levels were significantly lower compared to before the treatment $(p<0.0001)$. HDL cholesterol was significantly increased and TG was significantly decreased after SGLT2 inhibitor treatment. eGFR was significantly decreased and Crn was significantly increased, but no case became a problem clinically such as the development of renal failure. These data suggest that SGLT2 inhibitors exert beneficial effects on various clinical parameters including body weight, glucose metabolism, and liver injury.

To examine the effects of SGLT2 inhibitor on body composition, we evaluated it by using InBody 770 . Visceral fat area was significantly decreased from baseline $\left(140 \mathrm{~cm}^{2}\right.$ at baseline and $130 \mathrm{~cm}^{2}$ at 12 weeks, $\left.p<0.0001\right)$. Skeletal muscle mass was also significantly reduced from baseline, but the change was smaller than that of visceral fat area. These data suggest that SGLT2 inhibitors exert beneficial effects on body mass composition; SGLT2 inhibitors could reduce fat mass without substantial reduction of skeletal muscle mass, leading to amelioration of glycemic control.

The number (\%) of side effects in this study was as follows: hypoglycemia, $n=2(1.3 \%)$; abnormal appetite, 9 (5.8\%); dehydration, 1 $(0.6 \%)$; thirstiness, 5 (3.2\%); genitourinary tract infection, 4 (2.6\%); pruritus vulvae, 7 (4.5\%); cutaneous symptom; 3 (1.9\%). 
Table 1 Clinical parameters of all subjects in this study at baseline and 12 weeks after SGLT2 treatment

\begin{tabular}{|c|c|c|c|c|c|}
\hline \multirow[t]{2}{*}{ Clinical parameter } & \multicolumn{2}{|l|}{ Baseline } & \multicolumn{2}{|c|}{12 weeks } & \multirow[b]{2}{*}{$p$} \\
\hline & Median & Interquartile range & Median & Interquartile range & \\
\hline Body weight (kg) & 78.5 & $69.0-92.5$ & 75.7 & $67.2-91.4$ & $<0.0001$ \\
\hline BMI $\left(\mathrm{kg} / \mathrm{m}^{2}\right)$ & 29.6 & $25.9-34.3$ & 28.7 & $25.2-32.9$ & $<0.0001$ \\
\hline Visceral fat area $\left(\mathrm{cm}^{2}, n=108\right)$ & 140 & $102-79$ & 130 & $92-174$ & $<0.0001$ \\
\hline Skeletal muscle mass $(\mathrm{kg}, n=108)$ & 26.7 & $22.4-31.6$ & 26.6 & $22.2-31.1$ & $<0.0005$ \\
\hline HbAlc (\%) & 7.5 & $6.9-8.4$ & 7.1 & $6.6-7.7$ & $<0.0001$ \\
\hline Fasting PG $(\mathrm{mg} / \mathrm{dL}, n=87)$ & 138 & $120-158$ & 123 & $110-144$ & $<0.0001$ \\
\hline Postprandial PG (mg/dL, $n=28)$ & 186 & $150-236$ & 159 & $123-196$ & $<0.01$ \\
\hline Urinary glucose (g/g Crn) & 0.07 & $0-0.39$ & 43.78 & $24.36-66.30$ & $<0.0001$ \\
\hline HOMA- $\beta(n=61)$ & 60.7 & $30.0-80.7$ & 63.0 & $39.6-98.0$ & NS \\
\hline HOMA-IR $(n=61)$ & 4.1 & $2.6-5.8$ & 3.1 & $1.8-5.4$ & $<0.0005$ \\
\hline LDL cholesterol (mg/dL) & 100 & $81-118$ & 99 & $77-117$ & NS \\
\hline HDL cholesterol (mg/dL) & 47 & $39-54$ & 48 & $42-56$ & $<0.0001$ \\
\hline Non-HDL cholesterol (mg/dL) & 125 & $106-141$ & 124 & $103-142$ & 0.12 \\
\hline $\mathrm{TG}(\mathrm{mg} / \mathrm{dL})$ & 129 & $87-182$ & 115 & $77-162$ & $<0.05$ \\
\hline $\operatorname{NEFA}(\mu \mathrm{Eq} / \mathrm{L}, n=70)$ & 685 & $491-881$ & 707 & $566-836$ & NS \\
\hline Systolic BP $(\mathrm{mmHg})(n=100)$ & 130 & $123-138$ & 130 & $120-139$ & NS \\
\hline Diastolic BP $(\mathrm{mmHg})(n=100)$ & 80 & $76-87$ & 81 & $74-88$ & NS \\
\hline $\operatorname{Crn}(\mathrm{mg} / \mathrm{dL})$ & 0.60 & $0.47-0.75$ & 0.62 & $0.52-0.75$ & $<0.0005$ \\
\hline $\mathrm{eGFR}\left(\mathrm{mL} / \mathrm{min} / 1.73 \mathrm{~m}^{2}\right)$ & 95.2 & $81.5-115.7$ & 90.0 & $80.8-108.4$ & $<0.0005$ \\
\hline Urinary albumin $(\mathrm{mg} / \mathrm{g}$ Crn, $n=108)$ & 22.6 & $10.4-69.1$ & 23.7 & $11.1-58.3$ & NS \\
\hline Uric acid $(\mathrm{mg} / \mathrm{dL})$ & 5.4 & $4.4-6.2$ & 4.8 & $3.9-5.5$ & $<0.0001$ \\
\hline $\operatorname{ALT}(\mathrm{U} / \mathrm{L})$ & 34 & $21-55$ & 28 & $18-42$ & $<0.0001$ \\
\hline $\operatorname{AST}(\mathrm{U} / \mathrm{L})$ & 26 & $20-39$ & 24 & $18-34$ & $<0.0001$ \\
\hline$\gamma-\mathrm{GTP}(\mathrm{U} / \mathrm{L})$ & 38 & $23-67$ & 30 & $19-51$ & $<0.0001$ \\
\hline $\mathrm{ChE}(\mathrm{U} / \mathrm{L}, n=137)$ & 372 & $327-436$ & 364 & $325-421$ & $<0.01$ \\
\hline $\mathrm{CRP}(\mathrm{mg} / \mathrm{dL}, n=98)$ & 0.17 & $0.08-0.28$ & 0.15 & $0.07-0.29$ & NS \\
\hline
\end{tabular}

Data are median (interquartile range). $p$ values were calculated by Wilcoxon signed-rank test to compare between two paired groups. $p<0.05$ was considered significant

SGLT2 sodium-glucose co-transporter 2, BMI body mass index, HbA1c hemoglobin A1c, PG plasma glucose, HOMA- $\beta$ homeostasis model assessment of $\beta$-cell function, HOMA-IR homeostasis model assessment of insulin resistance, $L D L$ lowdensity lipoprotein, $H D L$ high-density lipoprotein, $T G$ triglyceride, $N E F A$ non-esterified fatty acid, $B P$ blood pressure, $C r n$ creatinine, $e G F R$ estimated glomerular filtration rate, $A L T$ alanine aminotransferase, $A S T$ aspartate transaminase, $\gamma-G T P \gamma$ glutamyltransferase, $C h E$ cholinesterase, $C R P$ C-reactive protein 
Evaluation of Factors Associated with Efficacy of SGLT2 Inhibitor on Liver
Damage

To examine which factors are associated with the effect of SGLT2 inhibitor on liver injury, we performed univariate analyses. In univariate analyses, we included the factors which may contribute to the improvement of liver injury or which had a significant change after SGLT2 treatment. As shown in Table 2, $\Delta$ ALT (from baseline to 12 weeks after the treatment) was significantly associated with $\mathrm{HbA1c}(p<0.001)$, fasting PG $(<0.05)$, IRI $(<0.05)$, HOMA-IR

Table 2 Association of baseline clinical parameters and alteration of ALT ( $\triangle$ ALT) for 12 weeks in univariate and multivariate analyses

\begin{tabular}{llllll}
\hline Parameter & $\boldsymbol{\rho}$ & $\boldsymbol{p}$ & Parameter & $\boldsymbol{\rho}$ & $\boldsymbol{p}$ \\
\hline Univariate analyses & & & & & \\
Age & 0.190 & $<0.05$ & LDL cholesterol & 0.117 & NS \\
Duration of diabetes & 0.196 & $<0.05$ & HDL cholesterol & 0.077 & NS \\
Body weight & -0.025 & NS & TG & -0.169 & NS \\
BMI & -0.084 & NS & NEFA & -0.143 & NS \\
Visceral fat area & -0.074 & NS & ALT & -0.552 & $<0.0001$ \\
Skeletal muscle mass & 0.165 & NS & AST & -0.494 & $<0.0001$ \\
HbAlc & -0.271 & $<0.001$ & $\gamma$-GTP & -0.262 & $<0.0005$ \\
Fasting PG & -0.216 & $<0.05$ & Crn & 0.228 & $<0.005$ \\
Postprandial PG & -0.113 & NS & eGFR & -0.187 & NS \\
IRI & -0.248 & $<0.05$ & Uric acid & -0.048 & NS \\
HOMA-IR & -0.296 & $<0.01$ & Urinary albumin & -0.044 & NS \\
CRP & -0.117 & NS & & &
\end{tabular}

\begin{tabular}{llll}
\hline Parameter & $\boldsymbol{\rho}$ & $\boldsymbol{F}$ & $\boldsymbol{p}$ \\
\hline Multivariate analyses & & & $\mathrm{NS}$ \\
Age & -0.014 & 0.02 & $\mathrm{NS}$ \\
Gender & -0.168 & 1.89 & $<0.05$ \\
HbAlc & -0.233 & 5.12 & $\mathrm{NS}$ \\
HOMA-IR & 0.183 & 3.17 & $\mathrm{NS}$ \\
Crn & 0.067 & 0.27 & $<0.001$ \\
ALT & -0.527 & 27.52 &
\end{tabular}

In analysis of the association between $\triangle$ ALT and various baseline clinical parameters, univariate analyses and multiple regression analyses were performed after Box-Cox Y conversion of the non-normal distributional variables

$A L T$ alanine aminotransferase, $B M I$ body mass index, $H b A I c$ hemoglobin Alc, $P G$ plasma glucose, IRI immunoreactive insulin, HOMA-IR homeostasis model assessment of insulin resistance, CRP C-reactive protein, $L D L$ low-density lipoprotein, $H D L$ high-density lipoprotein, $T G$ triglyceride, $N E F A$ non-esterified fatty acid, $A S T$ aspartate transaminase, $\gamma$ GTP $\gamma$-glutamyltransferase, Crn creatinine, eGFR estimated glomerular filtration rate, $N S$ not significant 
$(<0.05), \operatorname{ALT}(p<0.0001)$, AST $(p<0.0001), \gamma-$ GTP $(p<0.0005)$, and Crn $(<0.005)$ at baseline.

To examine which factors independently determine the effect of SGLT2 inhibitor on alteration of liver damage, we performed multivariate analyses using age, gender, baseline HbA1c, baseline HOMA-IR, baseline Crn, and baseline ALT as explanatory variables and $\Delta$ ALT (0-12 weeks) as an objective variable. It is noted that we included baseline ALT so that we excluded the possible influence of baseline ALT on this analysis. Baseline HbA1c and ALT were independently associated with $\Delta$ ALT (0-12 weeks). SGLT2 inhibitors exerted more beneficial effects on liver injury in subjects with high HbA1c and high ALT at baseline.
To examine the association between the alteration of liver damage and that of other parameters after SGLT2 inhibitor treatment, we performed univariate analyses. As shown in Table 3, $\Delta$ ALT (0-12 weeks) was significantly associated with $\Delta$ HbA1c $(p<0.0001), \Delta$ fasting PG $(p<0.05), \Delta$ AST $(p<0.0001)$, and $\Delta \gamma$-GTP $(p<0.0001)(0-12$ weeks $)$.

To examine which factors independently determine the effect of SGLT2 inhibitor on liver damage, we performed multivariate analyses using age, gender, and $\Delta \mathrm{HbA} 1 \mathrm{c}$ as explanatory variables and $\Delta$ ALT (0-12 weeks) as an objective variable. $\Delta$ HbA1c and age were independently associated with $\Delta$ ALT (0-12 weeks). SGLT2 inhibitors exerted more beneficial effects on

Table 3 Association of alteration of various clinical parameters and alteration of ALT ( $\Delta$ ALT) for 12 weeks in univariate and multivariate analyses

\begin{tabular}{|c|c|c|c|c|c|}
\hline Parameter & $\rho$ & $p$ & parameter & $\rho$ & $p$ \\
\hline \multicolumn{6}{|l|}{ Univariate analyses } \\
\hline$\Delta$ Body weight & 0.171 & $<0.05$ & $\Delta \mathrm{LDL}$ cholesterol & 0.023 & NS \\
\hline$\Delta \mathrm{BMI}$ & 0.185 & $<0.05$ & $\Delta \mathrm{HDL}$ cholesterol & -0.030 & NS \\
\hline$\Delta$ Visceral fat area & 0.179 & NS & $\Delta \mathrm{TG}$ & -0.015 & NS \\
\hline$\Delta$ Skeletal muscle mass & 0.042 & NS & $\Delta$ NEFA & 0.194 & NS \\
\hline$\Delta \mathrm{HbAlc}$ & 0.312 & $<0.0001$ & $\Delta \mathrm{AST}$ & 0.823 & $<0.0001$ \\
\hline$\Delta$ Fasting PG & 0.231 & $<0.05$ & $\Delta \gamma-\mathrm{GTP}$ & 0.630 & $<0.0001$ \\
\hline$\Delta$ Postprandial PG & -0.038 & NS & $\Delta$ eGFR & 0.154 & NS \\
\hline$\Delta$ IRI & 0.116 & NS & $\Delta$ Uric acid & -0.073 & NS \\
\hline$\Delta \mathrm{CRP}$ & 0.031 & NS & $\Delta$ Urinary albumin & 0.034 & NS \\
\hline Parameter & \multicolumn{2}{|c|}{$\rho$} & \multicolumn{2}{|l|}{$F$} & $p$ \\
\hline \multicolumn{6}{|l|}{ Multivariate analyses } \\
\hline Age & \multicolumn{2}{|c|}{0.174} & \multicolumn{2}{|l|}{4.71} & $<0.05$ \\
\hline Gender & \multicolumn{2}{|c|}{-0.095} & \multicolumn{2}{|l|}{1.57} & NS \\
\hline$\Delta \mathrm{HbAlc}$ & \multicolumn{2}{|c|}{0.244} & \multicolumn{2}{|l|}{9.22} & $<0.005$ \\
\hline
\end{tabular}

In analysis of the association between $\triangle \mathrm{ALT}$ and alteration of various clinical parameters, univariate analyses and multiple regression analyses were performed after Box-Cox Y conversion of the non-normal distributional variables

$A L T$ alanine aminotransferase, $B M I$ body mass index, $H b A 1 c$ hemoglobin Alc, $P G$ plasma glucose, IRI immunoreactive insulin, HOMA-IR homeostasis model assessment of insulin resistance, CRP C-reactive protein, $L D L$ low-density lipoprotein, $H D L$ high-density lipoprotein, $T G$ triglyceride, NEFA non-esterified fatty acid, $A S T$ aspartate transaminase, $\gamma$ GTP $\gamma$-glutamyltransferase, $e G F R$ estimated glomerular filtration rate, $N S$ not significant 
liver injury in younger subjects and/or in subjects whose HbA1c levels were decreased.

\section{Evaluation of Factors Associated with Efficacy of SGLT2 Inhibitor on Glycemic Control}

To examine which factors are associated with the effect of SGLT2 inhibitor on glycemic control, we performed univariate analyses. As shown in Table 4, $\Delta$ HbA1c (from baseline to 12 weeks after the treatment) was significantly associated with age $(p<0.0005)$, HbA1c $(p<0.001)$, postprandial PG $(p<0.05)$, HOMAIR $(p<0.05)$, NEFA $(p<0.05)$, ALT $(p<0.005)$, AST $\quad(p<0.005), \quad \gamma$-GTP $\quad(\mathrm{p}<0.005), \quad$ Crn $(p<0.005)$, and eGFR $(p<0.0005)$ at baseline.

To examine which factors independently determine the effect of SGLT2 inhibitor on glycemic control, we performed multivariate analyses using age, gender, and baseline levels of ALT, eGFR, HOMA-IR, NEFA, body weight, and $\mathrm{HbA1c}$ as explanatory variables and $\Delta$ HbA1c (0-12 weeks) as an objective variable. It is noted that we included baseline HbA1c so that we excluded the possible influence of baseline $\mathrm{HbA1c}$ on this analysis. Baseline HbA1c was independently associated with $\Delta \mathrm{HbA1c}$ (0-12 weeks). SGLT2 inhibitors exerted more beneficial effects on glucose tolerance in subjects with high HbA1c at baseline.

To examine the association between the alteration of $\mathrm{HbA} 1 \mathrm{c}$ and that of other parameters after SGLT2 inhibitor treatment, we performed univariate analyses. As shown in Table $5, \Delta$ HbA1c (0-12 weeks) was significantly associated with $\Delta$ body weight $(p<0.0005), \Delta$ BMI $(p<0.0005), \Delta$ fasting PG $(p<0.05), \Delta$ postprandial PG $(p<0.05), \Delta$ CRP $(p<0.01), \Delta$ NEFA $(p<0.01), \Delta$ ALT $(p<0.0001), \Delta$ AST $(p<0.001), \Delta \gamma$-GTP $(p<0.0001)$, and $\Delta$ eGFR $(p<0.01)$. To examine which factor independently determines the effect of SGLT2 inhibitor on glycemic control, we performed multivariate analyses using age, gender, duration, $\Delta$ BMI, $\Delta$ fasting PG, $\Delta$ NEFA, $\Delta$ eGFR, $\Delta$ ALT, and $\Delta$ CRP (0-12 weeks) as explanatory variables and $\Delta$ HbA1c (0-12 weeks) as an objective variable. In multivariate analyses, we included various factors which were associated with change of HbA1c in univariate analyses. $\Delta$ ALT was independently associated with $\Delta$ HbA1c (0-12 weeks). SGLT2 inhibitors exerted more beneficial effects on glycemic control in subjects whose liver injury was ameliorated.

\section{Evaluation of Factors Associated with Efficacy of SGLT2 Inhibitor on Body Weight}

To examine which factor determines the effect of SGLT2 inhibitor on body weight, we performed univariate analyses. There was no association, however, between $\Delta \mathrm{BMI}$ (from baseline to 12 weeks after the treatment) and any other parameters at baseline including age, duration, body weight, BMI, fasting and postprandial PG, IRI, HOMA-IR, LDL and HDL cholesterol, TG, NEFA, ALT, AST, $\gamma$-GTP, eGFR, uric acid, urinary albumin, or CRP.

To examine the association between the alteration of BMI and that of other parameters after SGLT2 inhibitor treatment, we performed univariate analyses. $\Delta$ BMI (0-12 weeks) was significantly associated with $\Delta$ body weight $(p<0.0001), \Delta$ visceral fat mass $(<0.0001), \Delta$ skeletal muscle area $(p<0.0001), \Delta$ ALT $(p<0.05), \Delta$ AST $(p<0.05)$, and $\Delta \gamma$-GTP and $\Delta$ eGFR $(p<0.005)(0-12$ weeks). There was no significant association between $\Delta \mathrm{BMI}$ and other parameters including $\Delta$ fasting PG, $\Delta$ postprandial PG, $\Delta$ IRI, $\Delta$ MOMA-IR, $\Delta$ CRP, $\Delta$ LDL cholesterol, $\Delta$ HDL cholesterol, $\Delta$ TG, $\Delta$ NEFA, $\Delta$ eGFR, $\Delta$ uric acid, or $\Delta$ urinary albumin.

To examine which factor independently determines the effect of SGLT2 inhibitor on body weight and BMI, we performed multivariate analyses using age, gender, $\Delta \mathrm{HbA1c}$, and $\Delta$ visceral fat area (0-12 weeks) as explanatory variables and $\Delta$ BMI $(0-12$ weeks) as an objective variable. In multivariate analyses, we included various factors which were associated with change of BMI in univariate analyses. $\Delta$ $\mathrm{HbA} 1 \mathrm{c}$ and $\Delta$ visceral fat area were independently associated with $\Delta$ BMI (0-12 weeks). SGLT2 inhibitors exerted more beneficial effects on BMI in subjects whose HbA1c levels and/or visceral fat area was decreased. 
Table 4 Association of baseline clinical parameters and alteration of HbAlc $(\Delta$ HbAlc) for 12 weeks in univariate and multivariate analyses

\begin{tabular}{llllll}
\hline Parameter & $\boldsymbol{\rho}$ & $\boldsymbol{p}$ & Parameter & $\boldsymbol{\rho}$ & $\boldsymbol{p}$ \\
\hline Univariate analyses & & & & & \\
Age & 0.281 & $<0.0005$ & LDL cholesterol & -0.102 & NS \\
Duration of diabetes & 0.153 & NS & HDL cholesterol & 0.152 & NS \\
Body weight & -0.197 & $<0.05$ & TG & -0.111 & NS \\
BMI & -0.186 & $<0.05$ & NEFA & -0.249 & $<0.05$ \\
Visceral fat area & -0.083 & NS & ALT & -0.229 & $<0.005$ \\
Skeletal muscle mass & 0.085 & NS & AST & -0.249 & $<0.005$ \\
HbAlc & -0.573 & $<0.001$ & $\gamma$-GTP & -0.249 & $<0.005$ \\
Fasting PG & -0.088 & NS & Crn & 0.229 & $<0.005$ \\
Postprandial PG & -0.398 & $<0.05$ & eGFR & -0.294 & $<0.0005$ \\
IRI & -0.183 & NS & Uric acid & 0.094 & NS \\
HOMA-IR & -0.236 & $<0.05$ & Urinary albumin & 0.010 & NS \\
CRP & -0.126 & NS & & & \\
\hline Paramer & & & & &
\end{tabular}

\begin{tabular}{llll}
\hline Parameter & $\boldsymbol{\beta}$ & $\boldsymbol{F}$ & $\boldsymbol{p}$ \\
\hline Multivariate analyses & & & NS \\
Age & 0.080 & 0.42 & NS \\
Gender & -0.092 & 0.58 & NS \\
ALT & -0.115 & 0.82 & NS \\
eGFR & 0.129 & 1.07 & NS \\
HOMA-IR & 0.074 & 0.27 & NS \\
NEFA & 0.124 & 1.18 & NS \\
Body weight & -0.093 & 0.45 & $<0.0001$ \\
HbAlc & -0.580 & 22.48 &
\end{tabular}

In analysis of the association between $\Delta \mathrm{HbAlc}$ and various baseline clinical parameters, univariate analyses and multiple regression analyses were performed after Box-Cox Y conversion of the non-normal distributional variables

$H b A 1 c$ hemoglobin A1c, BMI body mass index, $P G$ plasma glucose, IRI immunoreactive insulin, HOMA-IR homeostasis model assessment of insulin resistance, $C R P$ C-reactive protein, $L D L$ low-density lipoprotein, $H D L$ high-density lipoprotein, $T G$ triglyceride, NEFA non-esterified fatty acid, $A L T$ alanine aminotransferase, $A S T$ aspartate transaminase, $\gamma$ GTP $\gamma$-glutamyltransferase, $C r n$ creatinine, $e G F R$ estimated glomerular filtration rate, $N S$ not significant

\section{DISCUSSION}

In this study, we showed that SGLT2 inhibitor exerted beneficial effects on metabolic parameters such as body weight, HbA1c, and liver damage without severe adverse effects (Table 1). In addition, we evaluated the effect of SGLT2 inhibitor on body composition using 
Table 5 Association of alteration of various clinical parameters and alteration of HbAlc ( $\Delta$ HbAlc) for 12 weeks in univariate and multivariate analyses

\begin{tabular}{|c|c|c|c|c|c|}
\hline Parameter & $\rho$ & $p$ & Parameter & $\rho$ & $p$ \\
\hline \multicolumn{6}{|l|}{ Univariate analyses } \\
\hline$\Delta$ Body weight & 0.287 & $<0.0005$ & $\Delta \mathrm{LDL}$ cholesterol & 0.083 & NS \\
\hline$\Delta \mathrm{BMI}$ & 0.286 & $<0.0005$ & $\Delta \mathrm{HDL}$ cholesterol & 0.035 & NS \\
\hline$\Delta$ Visceral fat area & 0.103 & NS & $\Delta \mathrm{TG}$ & -0.148 & NS \\
\hline$\Delta$ Skeletal muscle mass & 0.194 & $<0.05$ & $\Delta$ NEFA & 0.323 & $<0.01$ \\
\hline$\Delta$ Fasting PG & 0.229 & $<0.05$ & $\Delta \mathrm{ALT}$ & 0.312 & $<0.0001$ \\
\hline$\Delta$ Postprandial PG & 0.391 & $<0.05$ & $\Delta \mathrm{AST}$ & 0.270 & $<0.001$ \\
\hline$\Delta \mathrm{IRI}$ & -0.106 & NS & $\Delta \gamma$-GTP & 0.385 & $<0.0001$ \\
\hline \multirow[t]{3}{*}{$\Delta \mathrm{CRP}$} & \multirow[t]{3}{*}{0.281} & $<0.01$ & $\Delta$ eGFR & 0.217 & $<0.01$ \\
\hline & & & $\Delta$ Uric acid & -0.138 & NS \\
\hline & & & $\Delta$ Urinary albumin & 0.076 & NS \\
\hline Parameter & \multicolumn{2}{|r|}{$\beta$} & \multicolumn{2}{|l|}{$\boldsymbol{F}$} & $p$ \\
\hline \multicolumn{6}{|l|}{ Multivariate analyses } \\
\hline Age & \multicolumn{2}{|r|}{-0.030} & \multicolumn{2}{|l|}{0.04} & NS \\
\hline Gender & \multicolumn{2}{|r|}{0.280} & \multicolumn{2}{|l|}{3.66} & NS \\
\hline Duration & \multicolumn{2}{|r|}{0.141} & \multicolumn{2}{|l|}{0.83} & NS \\
\hline$\Delta \mathrm{BMI}$ & \multicolumn{2}{|r|}{0.177} & \multicolumn{2}{|l|}{1.17} & NS \\
\hline$\Delta$ Fasting PG & \multicolumn{2}{|r|}{0.056} & \multicolumn{2}{|l|}{0.15} & NS \\
\hline$\triangle$ NEFA & \multicolumn{2}{|r|}{0.090} & \multicolumn{2}{|l|}{0.39} & NS \\
\hline$\Delta$ eGFR & \multicolumn{2}{|r|}{-0.053} & \multicolumn{2}{|l|}{0.12} & NS \\
\hline$\Delta \mathrm{ALT}$ & \multicolumn{2}{|r|}{0.339} & \multicolumn{2}{|l|}{4.67} & $<0.05$ \\
\hline$\Delta \mathrm{CRP}$ & \multicolumn{2}{|r|}{0.124} & \multicolumn{2}{|l|}{0.74} & NS \\
\hline
\end{tabular}

In analysis of the association between $\Delta \mathrm{HbAlc}$ and alteration of various clinical parameters, univariate analyses and multiple regression analyses were performed after Box-Cox Y conversion of the non-normal distributional variables HbAIc hemoglobin A1c, BMI body mass index, $P G$ plasma glucose, IRI immunoreactive insulin, HOMA-IR homeostasis model assessment of insulin resistance, CRP C-reactive protein, $L D L$ low-density lipoprotein, $H D L$ high-density lipoprotein, $T G$ triglyceride, $N E F A$ non-esterified fatty acid, $A L T$ alanine aminotransferase, $A S T$ aspartate transaminase, $\gamma$ GTP $\gamma$-glutamyltransferase, $e$ GFR estimated glomerular filtration rate, $N S$ not significant

InBody 770 and showed that body fat mass was substantially decreased and accompanied by a reduction of skeletal muscle mass (Table 1). Finally, we showed that SGLT2 inhibitor exerted larger efficacy on liver injury in younger subjects and/or in subjects whose HbA1c levels were decreased (Table 3). We assume that liver injury is relatively easily recovered in young subjects compared to elderly subjects. In addition, we showed that SGLT2 inhibitors exerted more beneficial effects on glycemic control in subjects whose liver injury was ameliorated 
(Table 5). We think that these result were reasonable for the following reasons. When liver injury is ameliorated with SGLT2 inhibitor, insulin resistance is usually decreased, which leads to the amelioration of glycemic control. In addition, liver damage such as steatosis is often induced by chronic hyperglycemia under diabetic conditions. Therefore, it is reasonable that when glycemic control is ameliorated with SGLT2 inhibitor, liver injury is recovered in subjects with type 2 diabetes.

As shown in Table 1, visceral fat area was more substantially decreased compared to the alteration of skeletal muscle mass; the reduction of body fat mass at 12 weeks was $-10 \mathrm{~cm}^{2}$ (from 140 to $130 \mathrm{~cm}^{2}$ ), whereas the reduction of skeletal muscle mass was only $-0.1 \mathrm{~kg}$ (from 26.7 to $26.6 \mathrm{~kg}$ ) (the respective rate of change is $-4.5 \%$ and $-0.9 \%, p=0.005)$. It has been thought that SGLT2 inhibitors could induce sarcopenia, especially in older and frail diabetic patients, but we think that we might not need to be afraid of sarcopenia provided we use SGLT2 inhibitors in an appropriate way by avoiding their prescription in older and frail diabetic patients. We think that SGLT2 inhibitors would be suitable for relatively young and obese subjects. It is noted here that in this study subjects were middle aged (median age, 54 years old) and relatively obese (median BMI, $29.6 \mathrm{~kg} / \mathrm{m}^{2}$ ) which was the main reason why there was no serious clinical problem such as sarcopenia. We think that the data obtained in this study would not be necessarily true for relatively aged and lean subjects. We assume that the alteration of body mass composition reduction could induce some clinical problems such as sarcopenia in the case of relatively aged and lean subjects. Furthermore, since baseline body fat mass is usually relatively small in aged and lean subjects, we assume that skeletal mass, rather than body fat mass, would be likely reduced with SGLT2 inhibitors, which would further increase the potential risk of some clinical problems such as sarcopenia.

We think that the present study with realworld data has a certain impact. First, in this study, we showed in detail which factor in the baseline parameters or which alteration of parameters after SGLT2 inhibitor treatment were related to the improvement of liver injury or glycemic control. We think that this point would be very informative and useful for clinicians when they start the treatment with an SGLT2 inhibitor. In addition, while it was shown that SGLT2 inhibitors ameliorated liver injury in subjects with NAFLD [21], the subjects in this study had relatively mild liver injury. Therefore, our present results suggest that SGLT2 inhibitors exert beneficial effects on body weight and liver injury even in subjects who have relatively mild liver injury without NAFLD. Furthermore, the number patients with type 2 diabetes and mild liver injury has been markedly increasing worldwide, and SGLT2 inhibitors have become internationally available. Therefore, we hope that our study will provide timely information about the effectiveness of SGLT2 inhibitors on glucose metabolism and liver damage in subjects with type 2 diabetes and liver injury.

There are limitations in this study. First, since the subjects in this study were outpatients, the results were influenced by diet and/or exercise in each subject. Second, although the control patients without SGLT2 inhibitors are required for comparison with the enrolled patients, in this study we performed various analyses only in subjects who initiated SGLT2 inhibitor treatment. Third, we failed to evaluate imaging results such as abdominal ultrasonography (US) and/or computed tomography (CT) scan, although we knew that some imaging was performed in 101 subjects (64.7\%) in this study in the past and 80 subjects were diagnosed as having hepatosteatosis. Some prospective study with a larger number of subjects and with an imaging inspection such as US and CT would be necessary to strengthen the idea obtained in our analyses. In addition, it is well known that there is much difference in characteristics between Japanese and Caucasian patients. For example, BMI in Japanese subjects is much smaller than that in Caucasians, and pancreatic $\beta$-cells in Japanese are much more vulnerable to hyperglycemia compared to those in Caucasians. Indeed, insulin secretory capacity is easily decreased under diabetic conditions in Japanese subjects. In contrast, insulin secretion is usually increased in order to compensate insulin resistance in Caucasians with obese type 2 diabetes [22]. Therefore, since all subjects in this study 
were Japanese, the data obtained in this study would not be necessarily true for Caucasians.

Taken together, SGLT2 inhibitors exert beneficial effects on metabolic parameters such as body weight, glycemic control, and liver injury without severe adverse effects in Japanese subjects with type 2 diabetes. In addition, there was close association between the effects of SGLT2 inhibitor on liver injury and those on glycemic control in subjects with type 2 diabetes.

\section{CONCLUSIONS}

Recovery of liver injury with SGLT2 inhibitor treatment was closely associated with their effects on glycemic control in Japanese subjects with type 2 diabetes.

\section{ACKNOWLEDGEMENTS}

We thank all participants in the study.

Funding. This research did not receive any specific grant from funding agencies in the public, commercial, or not-for-profit sectors. No funding or sponsorship was received for this study or publication of this article. The article processing charges were funded by the authors.

Authorship. All named authors meet the International Committee of Medical Journal Editors (ICMJE) criteria for authorship for this manuscript, take responsibility for the integrity of the work as a whole, and have given final approval for the version to be published.

Authorship contributions. Tomoe Kinoshita, Masashi Shimoda, Hideaki Kaneto researched data and wrote the manuscript. Junpei Sanada, Yoshiro Fushimi, Yurie Hirata, Shintaro Irie, Atsushi Obata, Tomohiko Kimura, Hidenori Hirukawa, Kenji Kohara, Fuminori Tatsumi, Shinji Kamei, Shuhei Nakanishi, Tomoatsu Mune, Kohei Kaku participated in discussion.

Disclosures. Hideaki Kaneto has received honoraria for lectures and received scholarship grants from Sanofi, Novo Nordisk, Eli Lilly,
Boehringer Ingelheim, Taisho Toyama Pharma, MSD, Takeda, Ono Pharma, Daiichi Sankyo, Sumitomo Dainippon Pharma, Mitsubishi Tanabe Pharma, Kissei Pharma, Astellas, Novartis, Kowa, Chugai, Japan Foundation for Applied Enzymology and A2 Healthcare. Kohei Kaku has been an advisor to, received honoraria for lectures from, and received scholarship grants from Novo Nordisk Pharma, Sanwa Kagaku Kenkyusho, Takeda, Taisho Pharmaceutical Co., MSD, Taisho Toyama Pharma., Astellas, Kissei Pharma., Mitsubishi Tanabe Pharma. Co., Ono Pharma. Co., Sumitomo Dainippon Pharma, Novartis, Mitsubishi Tanabe Pharma, AstraZeneca, Nippon Boehringer Ingelheim Co., Fujifilm Pharma Co., and Sanofi. Masashi Shimoda has received honoraria for lectures from AstraZeneca and Sanofi. Shuhei Nakanishi has received honoraria for lectures from AstraZeneca and Sanofi. Tomoe Kinoshita, Junpei Sanada, Yoshiro Fushimi, Yurie Hirata, Shintaro Irie, Atsushi Obata, Tomohiko Kimura, Hidenori Hirukawa, Kenji Kohara, Fuminori Tatsumi, Shinji Kamei and Tomoatsu Mune have nothing to disclose.

Compliance with ethics guidelines. All procedures performed in studies involving human participants were in accordance with the ethical standards of the institutional and/or national research committee and with the 1964 Declaration of Helsinki and its later amendments or comparable ethical standards. Informed consent was obtained by opt out.

Data availability. The data sets generated and/or analyzed during the current study are available from the corresponding author on reasonable request.

Open Access. This article is distributed under the terms of the Creative Commons Attribution-NonCommercial 4.0 International License (http://creativecommons.org/licenses/ by-nc/4.0/), which permits any noncommercial use, distribution, and reproduction in any medium, provided you give appropriate credit to the original author(s) and the source, provide a link to the Creative Commons license, and indicate if changes were made. 


\section{REFERENCES}

1. Kaku K, Watada H, Iwamoto Y, et al. Efficacy and safety of monotherapy with the novel sodium/glucose cotransporter-2 inhibitor tofogliflozin in Japanese patients with type 2 diabetes mellitus: a combined Phase 2 and 3 randomized, placebocontrolled, double-blind, parallel-group comparative study. Cardiovasc Diabetol. 2014;13:65-79.

2. Kim Y, Babu AR. Clinical potential of sodium-glucose cotransporter 2 inhibitors in the management of type 2 diabetes. Diabetes Metab Syndr Obes. 2012;5:313-27.

3. List JF, Woo V, Morales E, Tang W, Fiedorek FT. Sodium-glucose cotransport inhibition with dapagliflozin in type 2 diabetes. Diabetes Care. 2009;32:650-7.

4. Ohtake Y, Sato T, Kobayashi T, et al. Discovery of tofogliflozin, a novel C-arylglucoside with an O-spiroketal ring system, as a highly selective sodium glucosecotransporter 2 (SGLT2) inhibitor for the treatment of type 2 diabetes. J Med Chem. 2012;55:7828-40.

5. Tanizawa Y, Kaku K, Araki E, et al. Long-term safety and efficacy of tofogliflozin, a selective inhibitor of sodium-glucose cotransporter 2, as monotherapy or in combination with other oral antidiabetic agents in Japanese patients with type 2 diabetes mellitus: multicenter, open-label, randomized controlled trials. Expert Opin Pharmacother. 2014;15:749-66.

6. Zhang L, Feng Y, List J, Kasichayanula S, Pfister M. Dapagliflozin treatment in patients with different stages of type 2 diabetes mellitus: effects on glycaemic control and body weight. Diabetes Obes Metab. 2010;12:510-6.

7. Stumvoll M, Goldstein BJ, Haeften TW. Type 2 diabetes: principles of pathogenesis and therapy. Lancet. 2005;365:1333-46.

8. Maliha G, Townsend RR. SGLT2 inhibitors: their potential reduction in blood pressure. J Am Soc Hypertens. 2015;9:48-53.

9. Ferrannini E, Muscelli E, Frascerra S, et al. Metabolic response to sodium-glucose cotransporter 2 inhibition in type 2 diabetic patients. J Clin Invest. 2014;124:499-508.

10. Merovci A, Solis-Herrera C, Daniele G, et al. Dapagliflozin improves muscle insulin sensitivity but enhances endogenous glucose production. J Clin Invest. 2014;124:509-14.

11. Kaneto H, Obata A, Kimura $\mathrm{T}$, et al. Beneficial effects of SGLT2 inhibitors for preservation of pancreatic $\beta$-cell function and reduction of insulin resistance. J Diabetes. 2017;9:219-25.

12. Obata A, Kubota N, Kubota $\mathrm{T}$, et al. Tofogliflozin improves insulin resistance in skeletal muscle and accelerates lipolysis in adipose tissue in male mice. Endocrinology. 2016;157:1029-42.

13. Okauchi S, Shimoda M, Obata A, et al. Protective effects of SGLT2 inhibitor luseogliflozin on pancreatic $\beta$-cells in obese type 2 diabetic $\mathrm{db} / \mathrm{db}$ mice. Biochem Biophys Res Commun. 2016;470:772-82.

14. Shimo N, Matsuoka T, Miyatsuka T, et al. Shortterm selective alleviation of glucotoxicity and lipotoxicity ameliorates the suppressed expression of key $\beta$-cell factors under diabetic conditions. Biochem Biophys Res Commun. 2015;467:948-54.

15. Kaneto H, Obata A, Shimoda $\mathrm{M}$, et al. Promising diabetes therapy based on the molecular mechanism for glucose toxicity: usefulness of SGLT2 inhibitors as well as incretin-related drugs. Curr Med Chem. 2016;23:3044-51.

16. Zinman B, Wanner C, Lachin JM, et al. Empagliflozin, cardiovascular outcomes, and mortality in type 2 diabetes. N Engl J Med. 2015;373:2117-28.

17. Verma S, Garg A, Yan AT, et al. Effect of empagliflozin on left ventricular mass and diastolic function in individuals with diabetes: an important clue to the EMPA-REG OUTCOME Trial? Diabetes Care. 2016;39:e212-3.

18. Ferrannini E, Mark M, Mayoux E. CV protection in the EMPA-REG OUTCOME Trial: a "thrifty substrate" hypothesis. Diabetes Care. 2016;39:1108-14.

19. Kaku K, Lee J, Mattheus M, et al. Empagliflozin and cardiovascular outcomes in Asian patients with type 2 diabetes and established cardiovascular disease-results from EMPA-REG OUTCOME. Circ J. 2017;81(2):227-34.

20. Wanner C, Inzucchi SE, Lachin JM, et al. Empagliflozin and progression of kidney disease in type 2 diabetes. N Engl J Med. 2016;375:323-34.

21. Ito D, Shimizu S, Inoue $\mathrm{K}$, et al. Comparison of Ipragliflozin and pioglitazone effects on nonalcoholic fatty liver disease in patients with type 2 diabetes: a randomized, 24-week, open-label, active-controlled trial. Diabetes Care. 2017;40:1364-72.

22. Fukushima $M$, Suzuki $M$, Seino $Y$. Insulin secretion capacity in the development from normal glucose tolerance to type 2 diabetes. Diabetes Res Clin Pract. 2004;66:S37-43. 